in these papers was indirect. We wish to report here a more direct confirmation of the major hypothesis made in this theory.

The basic hypothesis is that the mechanical properties of such materials as paper, regenerated cellulose sheets and fibres, and ice are controlled by the number, $n$, of hydrogen bonds per unit volume of material subjected to strain, and by the potential energy, $U_{0}$, of these bonds. Thus the theory predicts that Young's modulus, $E$, for such materials is directly proportional to $n^{\frac{1}{3}}$. The following series of experiments provide data against which this predietion can be tested. A moderately beaten high $\alpha$-cellulose content eucalypt pulp was acetylated ${ }^{5}$ to various extents at two temperatures, $35^{\circ} \mathrm{C}$. and $45^{\circ} \mathrm{C}$.; the percentage of the total $\mathrm{OH}$ groups so acetylated will be referred to as the degree of substitution. Paper sheets were made under standard conditions from these pulps and their stress-strain relationships for simple tension determined under standard conditions of temperature and relative humidity.

If the modulus $E$ is in fact a function of the number of hydrogen bonds being strained, then a plot of $100\left(1-E / E_{0}\right)$ against degree of substitution, where $E_{\mathrm{o}}$ is the modulus for the virgin pulp, should give a unique relationship. The lower curve on Fig. 1 indicates that in fact this is so. However, as the theory under test predicts that $E$ is proportional to $n^{\frac{1}{3}}$, the relationship between $100\left(1-E^{3} / E_{0}{ }^{3}\right)$ and degree of substitution is of primary interest. Half the points on the upper curve represent a plot of these quantities. This relationship is consistent with the main tenet of the hydrogen bond theory, but verification is provided by the following argument.

The area under the stress-strain curve is the rupture energy (R.E.). If the theory is valid, then the rupture energy should, to a first approximation, be proportional to $(n / N) U_{0}$, that is, to $n$, where $N=$ Avogadro's number. Thus $\left.100[1-\text { R.E./(R.E. })_{0}\right]$ should, when plotted against degree of substitution, give the same curve as that given by $100\left(1-E^{3} / E_{\mathrm{o}}{ }^{3}\right)$. The other half of the points on the upper curve of the figure were calculated from the rupture energy,

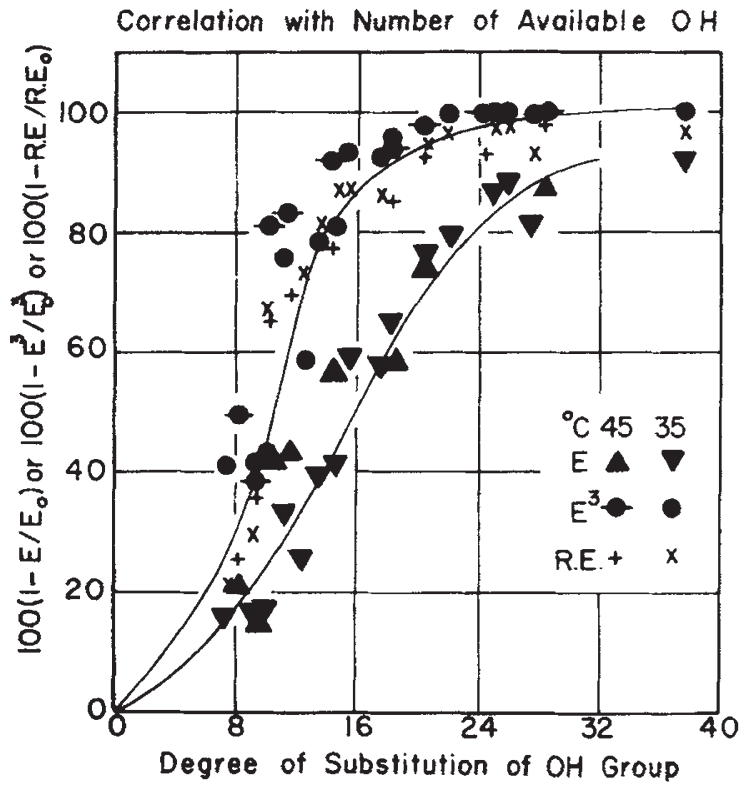

Fig. 1 and they appear to fall on the same curve as those calculated from $E^{3}$.

This curve deviates from the theory in two significant ways: (1) A linear relationship is predicted. It appears that the substitution of a hydrogen atom by a larger group not only reduces the number of hydrogen bonds but opens up the structure somewhat and, providing the process of substitution is not advanced, allows more bonds to form than would have been formed without the substitution. (2) It appears that the number of $\mathrm{OH}$ groups available for chemical substitution is larger than the number available for participation in those hydrogen bonds, within and between cellulose fibres, which can be deformed in the paper sheet. This is understandable as the $\mathrm{OH}$ groups at the surfaces of crystallites will be available for chemical reactions but will not contribute significantly to the mechanical properties, which are controlled by the amorphous fraction of the cellulose. Again, stress concentrations in the region of bonding between fibres may render the behaviour of hydrogen bonds so located of more significance during deformation than that of the bonds within the fibre. These and other details will be discussed elsewhere; the purpose of this letter is to demonstrate the validity of the basic hypothesis discussed.

Alfred H. Nissan

Department of Chemical Engineering,

Rensselaer Polytechnic Institute, Troy, N.Y.

H. G. Higains

Division of Forest Products, C.S.I.R.O.,

South Melbourne,

Australia

July 28.

${ }_{2}^{1}$ Nissan, A. H., Nature, 175, 424 (1955).

Nissan, A. H., Trans. Farad. Soc., 53, 700, 709 (1957).

Nissan, A. H., Nature, 178, 1411 (1956).

${ }^{4}$ Nissan, A. H., Tappi, 41, 131 (1958).

\section{Fibre Surface Replication by Rolling}

The communication by J. Mølgaard ${ }^{1}$ under the above heading invites the comment that this technique was developed at least seventeen years ago in the Wool Industries Research Laboratories in Leeds. Numerous applications in the study of the surface structure of wool fibres have been published by Dr. A. B. Wildman ${ }^{2}$ and the technique has recently attained text-book status ${ }^{3}$. I think it only fair to Dr. Wildman that his priority in this field should be acknowledged.

Textile Physics Laboratory,

$$
\text { H. J. Woods }
$$

Department of Textile Industries,

The University, Leeds. Sept. 30.

Molgaard, .., Nature, 184, 264 (1959).

Wildman, A. B., 'The Microscopy of Animal Textile Fibres' (Leeds, Wool Industries Research Association, 1954): Wildman, A. B., Proc. Int. Wool Textile Research Conf., Austratia, 1955, Vol. $F$,

p. 156.
Stoves, J. L., 'Fibre Microscopy', 187 (London: National Trade Press, 1957).

THE precedence of Dr. A. B. Wildman's technique, pointed out by Mr.H.J. Woods is freely acknowledged; I was unfortunately unaware of his work. The principles and effect of our procedure are the same as Dr. Wildman's, though details differ.

We are applying the technique to the study of synthetic fibre surfaces which show a finer structure than the animal fibre scale structure studied by 